\title{
The evolution of methods of diagnostics of male genitals tuberculosis
}

\author{
Abstract \\ The goal of the given research is to study the evolution of methods of male genitals \\ tuberculosis diagnostics in the last decades.
}

Keywords: Tuberculosis; male genitals; recombinant tuberculous allergen; sonography; targeted aspiration and biopsy; polymerase chain reaction; fibreurethrocystoscopy
Volume 6 Issue I - 2018

\section{Stepanov PI}

Urologist, Kiev regional anti-tuberculosis dispensary, Ukraine

\author{
Correspondence: Paul Stepanov, Candidate of Sciences, \\ Urologist, Kiev regional anti-tuberculosis dispensary, \\ Komsomolskaja, 23, Kiev region, city of Boyarka, Ukraine \\ Email profurolog@ukr.net
}

Received: November 16, 2017 | Published: January 23, 2018

\section{Abbreviations}

TB, tuberculosis; WHO, world health organisation; CR, chemoresistant; UGO, urogenital organs; ICD, international classification of diseases; MG, male genitals; PR, prostate; VS, vesicles; ARB, acidoresistant bacteria; PCR, polymerase chain reaction; ATAB, anti-TB antibodies; IFA, immune-fermental analysis; PSA: prostate specific blood antigen; TU, tuberculin units; CT: computer-tomography; CDM, colour doppler mapping; MRI, magnetic resonance imaging; MRT, magnetic resonance tomography

\section{Introduction}

In the last decades of the $20^{\text {th }}$ and $21^{\text {st }}$ centuries, a tuberculosis (TB) pandemic is remarkable in the whole world. According to data of the World Health Organisation (WHO), 9,0 M cases of TB diseases and $1,5 \mathrm{M}$ cases of death caused by TB were registered in $2014 .{ }^{1}$ According to WHO data, a TB epidemic took place in Ukraine since 1995. This is why TB has become one of the main problems of prior importance not only in Ukraine. According to data of the Ministry of Healthcare (Ukraine), the morbidity rate in Ukraine has declined by $20 \%$ in the last 7 years as a result of complex measures, and according to data on 2013 and 2014 it is less than $1 \%$ of the population, which means that we have succeeded in overcoming the TB epidemic. However, rates of morbidity, spreading and death remain at a high level. In 2014, 25543 TB diseased were detected in Ukraine, from which 13302 persons were bacteria-producing, which are the infection source. TB morbidity rate was 71.2 and the number of bacteria producers was 31.0 for $100 \mathrm{k}$ of population. ${ }^{2}$

At the present, the number of severe TB forms has increased, just as new phthisiology problems have emerged, one of which is increasing of chemo-resistant (CR) TB, co-infection: TB paired with HIV/AIDS. The highest number of HIV-infected and AIDS-diseased persons is observed among people aged 20-40 years [2-4]. According to data of the Ministry of Healthcare (Ukraine), 4441 patients with active TB and AIDS were detected in Ukraine in 2014, which was 10.4 for $100 \mathrm{k}$ of population, and the specific weight was $17.4 \%$ in the TB structure. ${ }^{2}$

Extra pulmonary TB localisations including those of urogenital organs (UGO) are a part of the general phthisiology problem. The increasing of TB spreading of respiratory organs must be accompanied by increasing of extra pulmonary TB frequency. ${ }^{1-3,5-11}$
The analysis of official statistical registration forms does not allow to have a full imagination of extra pulmonary TB localisations as the statistic registration forms only include the urogenital organs TB (A.18.1) - international classification of diseases 10 (ICD 10). There are no separations to TB of urine-producing organs and genitals of men and women. This is why a full personal examination must be performed to acquire a full image, as numerous publication authors point out. ${ }^{5-7}$

According to data of the Ministry of Healthcare (Ukraine), the extrapulmonary TB morbidity rate (A.15-18,19) was 5,7 for 100 $\mathrm{k}$ of population in 2014 , which was $8,0 \%$ in the TB structure. The urogenital organs TB morbidity rate (A.18.1) is on the fourth place in the extrapulmonary TB structure and is 0.3 for $100 \mathrm{k}$ of population (5.1\%), which is less than respiratory organs TB (A.15.4-9, A.16.3-9) $-58.7 \%$; bones and joints (A.18.0) - 17.1\%; peripherical lymph nodes (A.18.2) - 8.3\%. ${ }^{2}$

The structure of urological diseases among the population of Ukraine has recently faced substantial modifications - Inflammation diseases of urine-producing organs and genitals have gained the first place. ${ }^{12}$ They also include a specific inflammation UGO TB..$^{2,5,8,13,14}$

The given TB localisation problem is current, firstly because male genitals (MG) TB are quite widespread, but mainly scrotum organs TB are diagnosed. Prostate (PR) and vesicles (VS) TB is diagnosed significantly less frequently. ${ }^{5-25}$ Secondly, MG TB which was not detected on time entails a threat for life of the patient and his environment. Thirdly, MG TB diseased has a declined fertility only $3.1 \%$ could maintain it. This determines the social aspect of the problem, with a negative demographic situation not only in Ukraine. This is especially important for men in generative age with affection of one or both epididymitis, when even today, the main treating method is chirurgic (organ-removing): orchiectomy for $22.2 \%$ and epididymectomia for $45.5 \%$ of diseased, even unmarried, patients, which entails their sterilisation. ${ }^{4}$ Fourthly, this is a perturbation of the erectile and ejaculatory functions with MG TB, which is a severe negative moment in the social and family life activity not only of men. ${ }^{5}$

In the last years, a significant number of persistent ( $\mathrm{L}$ - forms) mycobacterium tuberculosis has emerged including preparations with anti-TB function resulting from wide application in general treating network (aminoglycosides, macrolides, fluoroquinolones etc.). This 
significantly aggravates the biological verification of mycobacterium tuberculosis. . $^{56-18,22,23,26-29}$ Currently, resulting from modifications of the immune status of human organism, for different reasons, specific characteristics of TB including MG TB are microscopically detected less and less frequently, which significantly aggravates its histological verification as well. ${ }^{5,11,14,15,18,20,21}$ For these reasons, the clinical disease image has modified as well, which significantly aggravates to perform a differentiated diagnostics and to determine a final MG TB diagnosis in early stages. ${ }^{5,17}$

Consequently, considering the named data, the morbidity rate and disease of MG TB makes the given problem not only medically, but also socially important.

\section{Research materials and methods}

An examination of 467 patients with MG TB aged 16 to 82 years took place (average age was $49 \pm 14$ years). Patients were subject to examination and treatment in the urogenital tuberculosis diseased department of the regional clinical tuberculosis hospital of Donetsk, Ukraine, for 25 years (from 1984 to 2008). From 2009 to 2013, MG TB was detected at 51 patients in the extrapulmonary TB department of the regional clinical tuberculosis hospital of Donetsk, Ukraine. In the last four years (from 2010 to 2013), MG TB was detected only at 17 diseased as in the last years, widespread and generalised TB forms are prevailing, frequently at patients with HIV/AIDS. In the background of TB affection of lungs, respiratory organs, bones and joints, lymph nodes, genitals are affected as well; as a rule, these are destructive forms with fistula generation. Statistically, these TB forms are not considered in the MG TB graphic.

Data of complaints, anamnesis (disease, life and epidemiological), physical examination including scrotum organs palpation and rectal finger examination was evaluated. ${ }^{5}$ Clinical, biochemical blood and urine analyses data were evaluated. Bacteriological examination of blood, urine, prostate secretion, seminal fluid included: Ziehl-Neelsen stain for acido-resistant bacteria (ARB), preparation on solid and liquid environments to detect mycobacterium tuberculosis growth. From 1997 on, molecular-genetic methods were applied, DNA was determined by means of a polymerase chain reaction (PCR) to verify mycobacterium tuberculosis and secondary flora and their sensitivity to preparations. $5,23,26-29$

Immunological examinations included the determination of antiTB antibodies (ATAB) in blood of sum fractions: $\operatorname{Ig} A+\operatorname{IgM}+\operatorname{IgG}$, by monoclonal antibodies to mycobacterium tuberculosis, by means of special test systems and applying immune-fermental analysis (IFA), from 1995 on. Also, from 1995 on, patients older than 40 years were determined their level of prostate specific blood antigen (PSA). ${ }^{5}$

Data of an immune-allergic test with 2 tuberculin units (TU) of purified tuberculin PPD-L until 2007 with subsequent application of recombinant TB allergen (Diaskintest ${ }^{\circledR}$ ) from 2008 on. ${ }^{30,31}$ Radiodiagnostics data was generated and evaluated as well:

A. X-ray examination methods: Overview radiography of thorax organs, urine-producing organs and lesser pelvis; excretion urography; cystography (descending and ascending); ascending urethrography, vesiculography, multi-spiral X-ray computertomography $(\mathrm{CT})$ of retroperitoneal space and lesser pelvis organs including $3 \mathrm{D}$ reconstruction. ${ }^{5}$

B. Radionucleide examination methods: Renography, kidneys scintigraphy and lymph nodes gamma scintigraphy. ${ }^{5}$
C. Sonographical examination methods: Kidneys, urine bladder with prostate, transcutane of scrotum organs as well as transrectal of prostate and seminal vesicles including blood stream examination applying colour Doppler mapping (CDM).

D. Magnetic resonance imaging (MRI) of retroperitoneal space, lesser pelvis organs, scrotum.

Application of transcutaneous and transrectal under sonographic control:

A. Aspiration of content: Hypoechogenic tissue areas, cysts, epididymis caverns, testicle, prostate and seminal vesicle to verify mycobacterium tuberculosis and secondary flora and their sensitivity to preparations (scopy, preparation, PCR);

B. Epididymis tissues biopsy, testicle, prostate and seminal vesicles membranes and bioptates examination applying classical histology, bioptate examination after Ziehl-Neelsen stain for acido-resistant bacteria and PCR for mycobacterium tuberculosis DNA.

C. Endoscopic examination methods were applied: urethra, urine bladder (hard: urethroscope and cystoscope), fibre-urethrocystoscopy to microscopically detect tubercles, subsequently taking their content to verify mycobacterium tuberculosis and secondary flora (scopy, preparation, PCR) and, if applicable: biopsy of urethra membrane, urine bladder and examination of bioptates applying classical histology, examination of bioptate after Ziehl-Neelsen stain for acido-resistant bacteria and PCR for mycobacterium tuberculosis DNA; catheterisation of ejaculatory ducts and seminal vesicles with aspiration of content to verify mycobacterium tuberculosis and secondary flora (scopy with Ziehl-Neelsen stain for acido-resistant bacteria, preparation on solid and liquid environments, PCR for DNA) (invention requested); if applicable, X-raying of contrast retrograde vesiculography.

\section{Research results}

Distribution of patients with MG TB according to age groups was: younger than 20 years: 19 (4.14\%) patients, from 21 to 30 years: 46 (9.85\%), from 31 to 40 years: $52(11.15 \%)$, from 41 to 50 years: 111 $(23.77 \%)$, from 51 to 60 years: $137(29.35 \%)$, from 61 to 70 years: 78 $(16.75 \%)$, from 71 to 80 years: $22(4.74 \%)$, and older than 80 years: $1(0.25 \%)$ (Figure 1).

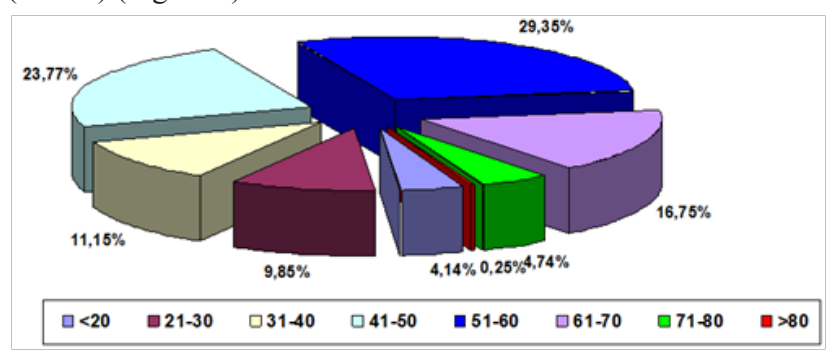

Figure I Patients with male genitals tuberculosis according to age groups.

The Mantoux test with 2 tuberculin units (TU) of purified tuberculin PPD-L to diagnose MG TB was applied for patients younger than 40 years until 2007. From 117 patients, 35 (29.9\%) had a positive one. From 2008 on, the recombinant TB allergen (Diaskintest ${ }^{\circledR}$ ) has been applied. From 21 patients, $18(85.7 \%)$ had a positive one.

Determination of anti-tuberculosis antibodies for mycobacterium tuberculosis was performed at 226 patients. 174 (76.9\%) patients 
showed to have summary antibodies for mycobacterium tuberculosis in blood, which allowed a screening for TB in patients including MG. The MG TB affection structure is shown in Tables 1-3.

Of patients with tubercle-infiltrated TB form of epididymis, the epididymis was enlarged, hard, tubercular, moderately painful when palpating. In sonography, the epididymis is enlarged; the structure is heterogenic due to areas with decreased echogenicity. In transcutaneous aspiration of tissue content in hypoechogenic areas under sonographic control and examination of the aspirate by PCR, mycobacterium tuberculosis DNA was detected, which allowed to avoid targeted biopsy of the epididymis. The same patients that were subject to transcutaneous biopsy under sonographic control all showed histological features of a TB granuloma in the biopsy material. $55(11.8 \%)$ men with a one-sided process were diagnosed an isolated tubercular-infiltrative epididymis TB (without TB affection of other genital system organs), 21 (4.5\%) patients were diagnosed a primary isolated tubercular-infiltrative epididymis TB (without TB affection of other organs and systems of the organism). In the biopsy material of the prostate of these patients, morphologic features of a non-specific prostatitis of toxico-allergic genesis was detected. None of the patients with epididymis TB and chronical and toxico-allergic prostatitis was detected acido-resistant bacteria in scopy with ZiehlNeelsen stain and mycobacterium tuberculosis growth on liquid and solid grounds. When examining the epididymis biopsy material by PCR, all examined persons were detected mycobacterium tuberculosis DNA. They were not detected when examining the prostate biopsy material by PCR.

Table I Structure of tuberculosis affection of scrotum organs with simultaneous involving of inner pelvic floor organs in the process, $\mathrm{n}(\%)$

\begin{tabular}{|c|c|c|c|c|c|c|c|}
\hline \multirow[t]{2}{*}{ Nosology } & \multirow[t]{2}{*}{ Total } & \multirow[t]{2}{*}{ One side } & \multirow[t]{2}{*}{$\begin{array}{l}\text { Both } \\
\text { sides+prostatitis }\end{array}$} & \multirow[t]{2}{*}{$\begin{array}{l}\text { Total } \\
\text { Prostatitis }\end{array}$} & \multicolumn{3}{|c|}{ Vesiculitis } \\
\hline & & & & & One & Both & \\
\hline Epididymitis & $162(34,7)$ & I03(22, I): & $59(12,6)$ & $107(22,9)$ & $24(23,3)$ & $67(41,3)$ & $91(5,2)$ \\
\hline Isolated epididymitis & $55(I I, 8)$ & Right $47(10,1)$ & +seminal vesicles & & & & \\
\hline \multirow[t]{4}{*}{ Initially isolated epididymitis } & $21(4.5)$ & Left 56(12,0) & $52(I I, I)$ & & & & \\
\hline & & \multicolumn{2}{|c|}{+ prostate $48(46,6)$} & & & & \\
\hline & & \multicolumn{2}{|c|}{ I seminal vesicle $24(23,3)$} & & & & \\
\hline & & \multicolumn{2}{|l|}{ Both I5 $(14,6)$} & & & & \\
\hline \multirow[t]{2}{*}{ Epididymoorchitis } & $198(42,4)$ & $132(28,2)$ & $66(14,1)$ & $186(39,8)$ & $50(10,7)$ & $138(29,5)$ & $188(40,3)$ \\
\hline & & \multicolumn{2}{|c|}{ Right and left respectively $66(14,1)$} & & & & \\
\hline Isolated epididymoorchitis & $12(2,6)$ & \multicolumn{2}{|c|}{ +prostate $120(25,7)$} & & & & \\
\hline $\begin{array}{l}\text { Epididymoorchitis at the left and } \\
\text { epididymitis at the right }\end{array}$ & $4(0,9)$ & - & $4(0,9)$ & $4(0,9)$ & - & - & - \\
\hline \multirow[t]{3}{*}{ Orchitis after epididymectomia } & $3(0,6)$ & Left $2(0,4)$ & - & $3(0,6)$ & & I $(0,2)$ & I $(0,2)$ \\
\hline & & \multicolumn{2}{|l|}{ Right I $(0,2)$} & & & & \\
\hline & & \multicolumn{2}{|l|}{ +prostate $3(0,6)$} & & & & \\
\hline \multirow[t]{2}{*}{ Total scrotum organs } & $372(79,7)$ & $238(50,9)$ & $134(28,7)$ & $305(65,3)$ & $74(15,8)$ & $206(44, I)$ & $280(59,9)$ \\
\hline & & \multicolumn{2}{|c|}{+ prostatitis $17 \mid(36,6)$} & & & & \\
\hline
\end{tabular}

Table 2 Structure of tuberculosis affection of scrotum organs without involving of inner pelvic floor organs in the process, $\mathrm{n}$ (\%)

\begin{tabular}{|c|c|c|c|}
\hline Nosology & Number & Prostatitis & Two-Sided Vesiculitis \\
\hline Patients with tuberculous prostatitis with spread urinary system tuberculosis & $66(14, I)$ & $66(14, I)$ & $5 I(10,9)$ \\
\hline Isolated tuberculous prostatitis with extra urogenital tuberculosis localisations & $27(5,8)$ & $27(5,8)$ & $10(2, I)$ \\
\hline Initially isolated tuberculous prostatitis at one of them & $15(3,2)$ & $15(3,2)$ & $12(2,6)$ \\
\hline Isolated seminal vesicles tuberculosis & I $(0,2)$ & & I $(0,2)$ \\
\hline Total & $94(20,1)$ & $93(19,9)$ & $74(15,8)$ \\
\hline
\end{tabular}

Table 3 Forms of tuberculosis affection of male genitals, $n(\%)$

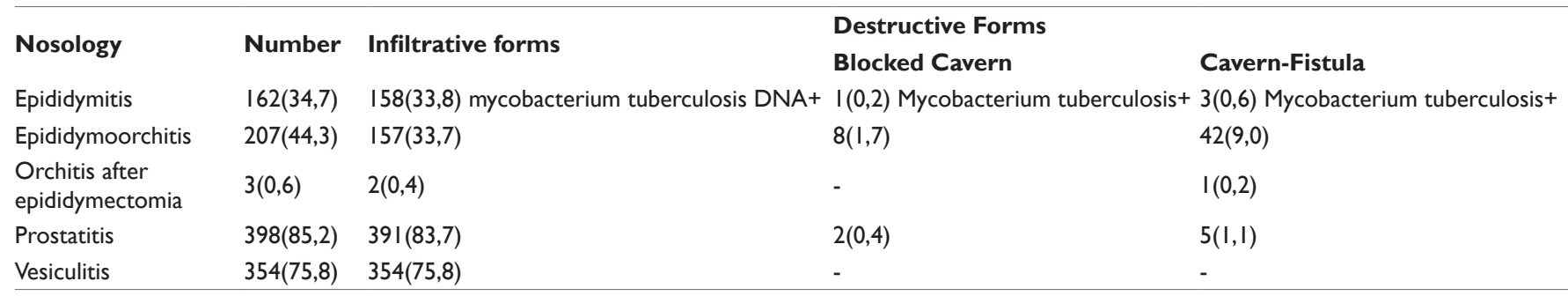


During palpation of the blocked-cavernous form epididymis, a tissue softening area involved in the inflammation process was detected. In sonography, a destruction cavity with uneven, thick, precious margins and heterogeneous content was detected. After needle aspiration of this content under sonographic control, it showed to have: acido-resistant bacteria in scopy with Ziehl-Neelsen stain, mycobacterium tuberculosis growth when preparing on solid and liquid environments, mycobacterium tuberculosis DNA in moleculargenetic examination. With a drained epididymis cavern, when examining the fistula excrete, the following was detected as well: acido-resistant bacteria, mycobacterium tuberculosis growth and mycobacterium tuberculosis DNA by PCR.

All of the 372 diseased with scrotum organs TB showed involving of vas deferens on the affected side or on both sides in the specific process.

$16(3.4 \%)$ men were detected a reactive hydrocele. All of these diseased were performed an operative correction: the hydrocele was removed. When examining the acquired liquid, none of them was detected mycobacterium tuberculosis by all 3 methods (scopy, preparation, PCR).

In simultaneous epididymis and testicle TB affection, firstly, the epididymis is involved in the process, then the second testicle if the inflammation progresses (Tables $1 \& 3$ ). Palpation and sonography data was analogue to modifications shown in the epididymitis TB, with the only difference that by palpation and sonography, the testicle was involved in the process as well. At tubercular-infiltrative TB form of epididymoorchitis, biopsy was performed under sonographic control of the epididymis as well as of the testicle parenchyma. Morphologic TB features was also detected in the epididymis and in the testicle. When examining the epididymis and testicle bioptates by PCR, mycobacterium tuberculosis DNA was also detected in all samples. In Ziehl-Neelsen stain of the bioptates, acido-resistant bacteria were detected in $25 \%$ of the samples. 12 (2.6\%) men with one-sided process were detected isolated epididymis and testicle TB. The prostates of these patients were also detected morphologic features of non-specific prostatitis of toxico-allergic genesis. The destructive epididymoorchitis TB form in aspirates of content of blocked caverns and in fistula excrete, the following was detected: acido-resistant bacteria, mycobacterium tuberculosis growth and mycobacterium tuberculosis DNA by PCR.

At the same time, we assume that epididymis and testicle TB is often combined with affection of other genital system organs. This is related firstly to the fact that male genitals are an entity of system: testicles, epididymis, vas deferens, vesicles, ejaculatory ducts, prostate, urethra with penis, are connected to each other by neuro-vessel, lymphatic paths and shared parotid ducts. The inflammation process in each of the named organs spreads to the neighbour organs and affects them in one or another level by the same pathologic process. This is why in each concrete case, it is necessary to verify the diagnosis of each male genital, histologically as well as for mycobacterium tuberculosis. ${ }^{26}$

The affection of internal pelvic genitals by TB at men with scrotum organs TB is shown in tables 1 и 3 .

Patients with tubercular-infiltrative TB form of the prostate were determined painful areas with increased thickness in the prostate during rectal finger examination. From the X-raying point of view, it was not possible to diagnose this prostate TB form. During transrectal sonography, areas of decreased echogenicity in prostate tissues were determined. During transrectal aspiration of the tissue content in hypoechogenic areas under sonographic control and aspirate examination by PCR, mycobacterium tuberculosis DNA was detected, which allowed to avoid targeted prostate biopsy. The patients that were subject to transrectal biopsy under sonographic control were all detected histologic TB granuloma features in the biopsy material.

Patients with destructive prostate tuberculosis forms showed painful areas of softened gland tissues in the prostate in rectal finger examination. In the period when sonography was impossible, $\mathrm{X}$-ray examinations were performed. Plan radiography of the pelvis area detected areas with calcinosis at the location of the former TB destruction focuses. In this case, numerous shadows of calcinosis areas, focus and asymmetry of their location were the characteristic features of TB. In case of draining prostate caverns into the urethra, $\mathrm{X}$-ray contrast urethra examination showed to be quite effective: ascending or descending urethrography. For initial prostate TB phases, retrograde contrasting of its flows in the form of a lace net, minor thread drains into the tissue were characteristic. When the process progressed, major drains of the contrast substance resulting from significant prostate destruction emerged. Destructive process can entail full prostate destruction with creation of a "pre-bladder". For initial modifications, filling of smooth-membrane cavities with the contrast substance is characteristic. In rare cases, draining of $\mathrm{X}$-ray contrast preparation in vas deferens and vesicles was observed. In sonography, destruction cavities with uneven, precise margins and heterogenic content were detected by a rectal measuring device. In needle aspiration of the content of the blocked cavern, under transrectal sonography control as well as in patients with draining caverns, in urine, excretion of fistula examination at the perinea, the following was detected: acido-resistant bacteria, mycobacterium tuberculosis growth and mycobacterium tuberculosis DNA by PCR

It is hardly possible to palpate the vesicles in the initial disease phase in patients with vesicles TB during finger rectal examination. When the prostate and the vesicles affected by TB are enlarged and welded with each other, it is possible to palpate painful conglomerates in vesicle projection during rectal examination.

In the period when sonography was impossible, X-ray examinations were performed as well. During vesiculography X-raying production, when after open excretion of the vas deferens and catheterisation, after the section or paracentesis of its lumen, X-ray contrast substance was injected, we found it productive to divide the detected modifications in certain and probable for prostate and vesicles TB diagnosis. The first ones included drains of contrast substance in the prostate tissue in case of a cavern connected to ejaculatory ducts visible on the photographies, the second ones included different X-ray features of a specific as well as of a non-specific vesicles affection of vas deferens and ejaculatory ducts. These are firstly narrowings and deformations of the vesicle lumen of different levels, missing of contrast of one or both vesicles, narrowing and obstruction of the vas deferens, missing of contrast of the ejaculatory duct. Narrowing and deformation of the vesicles lumen in diseased with urine-producing organs and genitals sometimes reached a significant expression level, and they acquired the appearance of a "burned tree". This feature was detected in $22.3 \%$ of the diseased. $8.3 \%$ of patients were detected missing contrast of vesicles or its amputation. It is much more frequently (36.1\% of diseased) that less rough modifications in vesicles in form of uneven contours, eroded or garland-like margins, unequal filling of the lumen when retentionally widened areas are paired with its narrowings or constrictions. $11 \%$ of diseased had vesicles dislocated to the side what directly depends on the prostate configuration. Blocked vas deferens at the affected epididymis side is detected in $28.1 \%$ of the diseased, 
from which are $14.1 \%$ of pelvis section, $14.1 \%$ also of groin and scrotum. In TB of both epididymis', the latter was observed in $37.9 \%$ of patients.

Vas deferens pathology was detected in $25 \%$ of patients, from which $11.2 \%$ were observed flow obstruction in pelvis section, what showed at vesiculograms by its abruption and traces of X-ray contrast substance beyond its borders. $66.5 \%$ of patients had a blurry contrasted vas deferens, with features of single or multiple narrowings over a period of time, $22.3 \%$ had a deformation and erosion of flow contours in its ampule.

From 236 patients with active urogenital organs TB who had undergone vesiculography, $63.8 \%$ suffered from scrotum organs TB. Two-sided vesiculography was performed at $74.3 \%$ of diseased. Most expressed modifications in vesicles and vas deferens corresponded to the affected side. Missing contrast of ejaculatory ducts was detected at vesiculographies at $72.2 \%$ of the examined. As a rule, they were observed some widening of the vesicle columns of retentional nature. However, the vas deferens remains passable as the vesicle was widened. As the vas deferens runs in the prostate layer, its pathology detected at vesiculograms has certain significance in prostate state evaluation. Missing contrast of the vas deferens can be evidence either for its cicatrisation or for compression of inflammatory modified prostate parenchyma. At the same time, the statement that the gland in always affected by TB in such cases is not justified as the inflammation process of any genesis in it can entail tissue infiltration, and consequently, flow compression.

X-ray computer tomography, magnetic resonance tomography as well as sonography have for the first time allowed acquiring an image of the prostate and vesicles without contrasting them. X-ray computer tomography of the prostate with TB was performed at 10 diseased; however, the data on the structure of the gland parenchyma was not precise like in sonography, and hence it was no longer applied for prostate TB diagnosis.

To diagnose involving of vesicles in the pathologic process, $\mathrm{X}$-ray computer tomography was performed at 14 diseased with male genital TB and functional perturbations of vesicles, hemato- and pyospermia, reduction of ejaculate volume, erectile dysfunction. 4 patients showed two-sided TB vesiculitis at the background of nephron-TB with involving the urine bladder in the process. 10 men were detected vesicle TB at the background of scrotum organs TB. From which: Two-sided vesiculitis was detected in 8 patients (4 of them with affected scrotum organs at both sides, and 2 at one side), and onesided at 4 men. X-ray computer tomography examination detected enlarged vesicles, more at the affected scrotum organs side. All of the other cases of two-sided TB vesiculitis showed asymmetrical vesicle affection - some vesicle was always more involved in the pathologic process. The structure of the content of vesicles was heterogenic: Areas of microcalcification and decreased density were detected. Pelvis cellulose showed single lymph nodes up to $0.8 \mathrm{~cm}$ in diameter. However, X-ray computer tomography also bears radiation stress for the diseased organism, which significantly limited its application for vesicles examination.

Transrectal sonography detected widened vesicles with infiltrated membranes and non-homogeneous content. In a one-sided process the modifications were asymmetric. Sonographic vesicles examinations were performed before and after ejaculation, which allowed to detect their missing emptying in affection by TB. In examination of the vesicles content after needle aspiration, mycobacterium tuberculosis DNA was detected by PCR, and after biopsy of their membranes, specific histological modifications were detected.
Also, patients with suspicion of vesicle TB were subject to fibrourethrocyctoscopy with simultaneous catheterisation of ejaculatory ducts and vesicles. After that, their content was aspirated with concluding mycobacterium tuberculosis and secondary flora verification (scopy, preparation, PCR for mycobacterium tuberculosis DNA).

From 467 examined patients, 94 (20.3\%) were diagnosed inner pelvic genitals TB without involving of scrotum organs in the process (Tables $2 \& 3$ )

Isolated (without involving of urine-producing and scrotum organs in the specific inflammation process) prostate TB was detected in 27 (5.8\% of $467 \mathrm{men}$ ), 11 of them were detected lungs TB in anamnesis, one was detected hip TB. 15 (55.5\%) diseased 27 patients were detected an initially isolated prostate TB (without affection of other organs and systems in the male organism by TB), which made up $3.2 \%$ of 467 examined persons. Two-sided vesicles TB was detected in 22 (81.4\% of 27 patients), which made up $4.7 \%$ of all 467 examined diseased persons (Table 2).

One patient with prostate TB of tubercle-infiltrative form had also an infiltrative form of TB of the hanging part of the urethra and an ulcer form of TB of the glans penis, which was morphologically proven.

One $(0.2 \%)$ man was detected isolated (without involving of other urine-producing and genital system organs in the specific inflammatory process) vesicles TB at the background of cavern TB of the upper part of the left lung with its following resection in the anamnesis

Consequently, from 467 examined patients, prostate TB was detected in $398(85.2 \%)$ men in total: of tubercle-infiltrative form in $391(83.7 \%)$, of destructive forms in $7(1,5 \%)$ : of cavern form in 2 $(0.4 \%)$, and cavern-fistula form in $5(1.1 \%)$ of the diseased. Vesicle TB was detected in $354(75,8 \%)$ : one-sided in $74(15.8 \%)$ and twosided in $280(60.0 \%)$ of the diseased (Tables $1-3)$.

One $(1.1 \%)$ patient of 94 without TB affection of scrotum organs had penis and scrotum skin TB at the penis base, which was morphologically proven, of a productive form with ulceration. A detailed examination of other TB focuses showed no pathology in the patient's organism. This made up $0.2 \%$ of all 467 examined diseased persons (Table 2).

\section{Discussion}

The MG TB diagnostic algorithm was subject to significant modifications in the past decades. Classic diagnostic methods, such as complaints clarification, anamnesis precision, physical examinations: examination and palpation of scrotum organs, finger rectal examination of prostate and vesicles remain unchanged.

Clinical and biochemical lab analyses of biological materials: blood, urine, genital glands secretions, fistula excretions remain unchanged as well.

TB tuberculinodiagnistics including MG TB was recently subject to significant modifications. Purified PPD-L tuberculin has stopped to response to antigenic features of modern mycobacterium tuberculosis, and hence the Mantoux reaction with $2 \mathrm{TU}$ as well as performance of diagnostic provocative tuberculin tests is not applied at the present. Instead of purified PPD-L tuberculin, TB recombinant allergen, (Diaskintest ${ }^{\circledR}$ ), is now applied for diagnostics, whose sensitivity is $85.7 \%$ according to our data 
Immunological examinations were subject to significant modifications. The blast-transformation leucocyte reaction and the neutrophils damage rate used in the precedent decades are practically not used at the present. At the present, anti-TB antibodies determination in blood according to monoclonal mycobacterium tuberculosis antibodies by ELISA is widely used, which allows to perform a screening on TB in patients, including MG TB. Also, if patients have a combination of TB and HIV/AIDS immunophenotyping methods with determination of the number of CD4, CD8 cells, and examination of other surface T-lymphocytes markers are widely used.

Mycobacterium tuberculosis verification methods were subject to important modifications. Only the method of examination of biological materials by microscopy with Ziehl-Neelsen stain for acido-resistant bacteria remains unchanged. This method has also started to be applied in examination of tissues bioptates, especially for patients with a combination of TB and HIV/AIDS. Data of preparation of biological materials on solid and liquid nutrient environments to verify mycobacterium tuberculosis in patients with MG TB are frequently negative and require a long time to acquire a reply. This is why at the present, the first place among mycobacterium tuberculosis verification methods was occupied by molecular-genetic examinations of biological materials by PCR to detect mycobacterium tuberculosis DNA and secondary flora. PCR is a rapid, precise and very specific mycobacterium tuberculosis verification method. All types of biological material are subject to this examination: urine, seminal fluid, fistula excretion, aspirates and bioptates from $\mathrm{MG}$ tissues.

MG TB radiation diagnostics methods were subject to substantial modifications. From X-ray methods, only plan radiography of thorax organs is applied at the present. Plan radiography of lesser pelvis organs, cystography (descending and ascending), ascending urethrography, vesiculography, multi-slice X-ray computer tomography of lesser pelvis organs are not used due to significant radiation stress. The leading place for MG examination is now firmly occupied by safe sonographic examinations: kidneys, urine bladder with prostate, scrotum organs: transcutaneous, prostate and vesicles: transrectal. Sonography allows identifying a hypo and hypoechogenic generation in MG. Sonography has allowed to detect missing emptying of vesicles when they are TB-affected.

Now, these are a possibility to perform under sonographic control targeted transcutaneous and transrectal aspiration of content of: hypoechogenic tissue areas, cysts, epididymis caverns, testicles, prostate and vesicles to verify mycobacterium tuberculosis and secondary flora as well as biopsy of epididymis, testicles, prostate and vesicle membranes.

Magnetic resonance tomography (MRT) ad lesser pelvis and scrotum organs is used less frequently at the present.

Examination of MG tissues bioptates is now performed not only with use of classic histology, but, as mentioned above, tissues microscopy for acido-resistant bacteria after Ziehl-Neelsen stain and molecular-genetic tissues examination for mycobacterium tuberculosis DNA and secondary flora.

Also, endoscopic examinations are now applied: of urethra, urine bladder by fibrourethrocystoscope, to microscopically detect tubercles with consequent obtaining of their content to verify mycobacterium tuberculosis and secondary flora, and, if applicable, perform biopsy as well as catheterisation of ejaculatory ducts and vesicles and aspiration of content to verify mycobacterium tuberculosis and secondary flora.

\section{Conclusion}

Hence, a diagnostics methods evolution has taken place, when some methods are now history of past and not applied any longer, and instead, other safer methods are used now for patients as well as for medical staff, and significantly more precise ones.

\section{Acknowledgements}

The study was financed by the regional clinical tuberculosis hospital and National medical University named Maxim Gorky of Donetsk, Ukraine.

\section{Conflicts of interest}

None.

\section{References}

1. Global tuberculosis report. WHO library cataloguing-in-publication data. 2014.

2. The incidence of tuberculosis and the activity of anti-TB institutions of Ukraine for the year 2014. Statistical Handbook DZ, Center of medical statistics of $\mathrm{MOH}$ of Ukraine, Ukraine.

3. Feshchenko YI. The Situation with tuberculosis in Ukraine. Doctor Phys for the practical doctors. 2002;4:11-14.

4. Unified clinical Protocol of primary, secondary (specialized) and tertiary (highly specialized) medical care Tuberculosis. The order of MH of Ukraine, pp. 620.

5. Kamyshan IS, Fedun ZV, Stepanov PI. Tuberculosis of the genital organs of men and women. Donetsk Express. 2002;278.

6. Kulchavenya EV, Krasnov VA, Skornyakov SN. Modern trends in epidemiological situation of tuberculosis watercannon. Tuberculosis and lung disease 12: 34-38.

7. Nechaeva OB, Skachkov VV. The Epidemiological situation of extrapulmonary tuberculosis in the Russian Federation. Tuberculosis and lung diseases. 2013;8:3-10.

8. Pavlova LP, Kamyshan IS, Saidakova NO, et al. Tuberculosis of the genitourinary organs of the Ukrainian population (condition, prognosis, causes reactivation treatment). Urology. 2001;3:15-29.

9. Buchholz NP, Salahuddin S, Haque R. Genitourinary tuberculosis: a profile of 55 in-patients. J Paк Med Assoc. 2000;50(8):265-269.

10. Gilbert J Wise, Venkata K Marella. Genitourinary manifestation of tuberculosis. Urologic Clinics of North America. 2003;30(1): 111-121.

11. Lee Y, Huang W, Huang J, et al. Efficacy of chemotherapy for prostatic tuberculosis-a clinical and histologic follow-up study. Urology. 2001;57(5):872-877.

12. Vozianov AF, Pasechnekov SP, Pavlova LP. Achievements and challenges of urological care to the population of Ukraine. Urology. $2001 ; 4: 3-6$.

13. Stepanov PI. Actual issues of pathogenesis of the lesions of the genital organs of men with tuberculosis. Urology. 2014;2:36-39.

14. Stepanov PI. Structure of the lesion of the genital organs of men with tuberculosis. Tuberculosis and lung diseases. 2014;3:49-56.

15. Brijatyuk EV, Kulchavenya EV, Baranchukova AA. Transrectal puncture biopsy of prostate under ultrasound guidance in the diagnosis of tuberculosis of the prostate. Innovative technologies in urology: Mat. VIII interregional. Scientific practical. Conf urologists of Siberia, pp. $2009 ; 120$. 
16. Kulchavenya EV. Difficulties in diagnosing tuberculosis of the genitourinary system. Novosibirsk. 2004.

17. Kulchavenya EV, Holtobin DP. Differential diagnosis of tuberculosis of the genitourinary system. Tuberculosis and lung disease. 2013;7:2-8.

18. Stepanov PI. Ways of increase of efficiency of early diagnostics and difference diagnosis of patients with tuberculosis of the prostate. Mater. III Congress of the TB and pulmonol. Ukr pulmonol journal. 2003;2(40):364

19. Stepanov PI. Tuberculosis of the penis. Men's health. 2006;1:32.

20. Stepanov PI. Tuberculosis of the seminal vesicles. Men's health. 2006;2:100-104.

21. Stepanov PI. Isolated tuberculosis of the prostate. Men's health. 2013;2:63-65.

22. Bernaschina CP, Cabrera M, Cardona P, et al. Genitourinary tuberculosis: the importance of early diagnosis and management. Case presentation. Bol Asoc Med P R. 1994;86(10-12):75-80.

23. Dochviri TZ, Katsitadze VA, Khosiashvili GZ, et al. Detection of mycobacteria tuberculosis in patients with urogenital tuberculosis by PCR method. Georgian Med News. 2002;(119):14-17.

24. Gokce G, Kilicarslan H, Ayan S, et al. Genitourinary tuberculosis: a review of 174 cases. Scand J Infect Dis. 2002;34(5):338-340.
25. Orakwe JC, Okafor PI. Genitourinary tuberculosis in Nigeria; a review of thirty-one cases. Niger J Clin Pract. 2005;8(2):69-73.

26. Kamyshan IS, Geev YV, Stepanov PI. Method for the diagnosis of urogenital tuberculosis. Declarative patent for the invention UA N 33776. 2001.

27. Kamyshan IS, Stepanov PI, Zyablitsev SV. Role of polymerase chain reaction in diagnosis of tuberculosis of the urinary and male sexual organs. Urologiia. 2003;3:36-39.

28. Kamyshan IS, Mams FN, Saidakova NA, et al. Diagnosis of tuberculosis of the urinary and male sexual organs polymerase chain reaction. Urology. 2004;2:44-47.

29. Kamyshan IS, Mams AN, Stepanov PI. The possibility of express methods of diagnosis of renal tuberculosis. Probl Tuberculosis. 2004;9:39-43

30. Kiselev VI, Baranowski PM, Pupyshev SA. A New skin test for diagnosis of tuberculosis based on the recombinant protein ESAT-CFP. Molek med. 2008;4:28-34.

31. Kiselev VI, Baranowski PM, Rudykh IV, et al. Clinical trial of a new skin test "DIASKINTEST ${ }^{\circledR}$ " for the diagnosis of tuberculosis. Probl tubes and lung diseases. 2009;2:11-16. 